\title{
La crisis y la salud. ¿La salud en crisis?
}

\author{
Crisis and health. Health in crisis?
}

DrCs. Klaus Thielmann, ${ }^{\mathrm{I}}$ DrCs. José Illnait Ferrer ${ }^{\mathrm{II}}$

I Miembro correspondiente de la Academia de Ciencias de Cuba. La Habana, Cuba.

II Centro Nacional de Investigaciones Científicas. La Habana, Cuba.

\section{RESUMEN}

La crisis económica y financiera que envuelve al mundo, también afecta la salud, porque los servicios de salud, tal y como se practican hoy dependen cada día más del mercado. En estas circunstancias la salud es convertida en una mercancía que se encarece en la medida en que también lo hacen los productos dedicados al diagnóstico y tratamiento de las enfermedades. Ello genera, a su vez, desigualdad de acceso a la medicina entre grupos de países ricos y países pobres e igualmente entre los ciudadanos al interior de todos aquellos países sometidos a regímenes neoliberales, o constituyen una carga adicional para los países donde los servicios médicos son financiados por la sociedad. El reto de las enfermedades crónicas no transmisibles impone la necesidad de una estrategia proactiva y salutogénica, basada en el fortalecimiento de la salud a través de sus determinantes como contribución no solo a la sostenibilidad y racionalidad de los servicios médicos, sino también al acceso igualitario allí donde se requiera. Un proyecto denominado "Determinantes Individuales y Sociales de la Salud en la Medicina Familiar", se ha iniciado en La Habana Vieja aprovechando la vocación preventiva de la medicina cubana y la preparación de sus médicos en esta práctica y que pretende estudiar la factibilidad y los beneficios de esta estrategia médica.

Palabras clave: Determinantes, salud, crisis económica.

\section{ABSTRACT}

The global financial and economic crisis also affects health because the health services increasingly depend on the market nowadays. Health is considered a 
commodity and becomes expensive due to rising prices of diagnostic and therapeutic products and services. At the same time, this exacerbates the inequality of access to health care between rich and poor countries and among the citizens within countries of neoliberal economic orientation. Health care turns into an additional burden for countries where the medical health services are financed by the society as a whole. The growing impact of chronic non-communicable diseases calls for a proactive and health-oriented strategy based on the health improvement through its determinants as a contribution to the efficiency and sustainability of medical care services and to equal access wherever is required. A project called 'Individual and Social Health Determinants in Family Medicine' was started in Old Havana, which is intended to demonstrate the feasibility and benefit of a health driven medical strategy. It builds on the genuine preventive orientation of Cuban medical practice, education and training.

Key words: Determinants, health, economic crisis.

\section{INTRODUCCIÓN}

La pregunta del título suena retórica. Así es, intencionalmente, porque pretendemos llamar la atención sobre una realidad preocupante. Se trata, por una parte, del problema conceptual más grave de la medicina contemporánea y por otra, de la necesidad de aprovechar la crisis para promover la reorientación y los cambios necesarios.

Cuando hoy en día se habla de "la crisis", esto desde luego se refiere, ante todo, a la crisis financiera y económica global que envuelve al mundo en esta primera década del siglo XxI. "La crisis" es omnipresente, concierne a todas las facetas de la vida, a todas las personas, perturba los equilibrios sociales, las relaciones nacionales e internacionales, causa turbulencias por todas partes y se revela ante nuestros ojos, de manera impresionante, la dependencia que tiene el "mundo desarrollado" de condiciones económicas y financieras cada vez más distorsionadas. Por fin, en 2011, tres años después de la quiebra del sistema bancario en los EE. UU. y en los países más ricos de Europa, se entiende, que no se trata de una de las crisis cíclicas "regulares" del capitalismo, sino del fracaso del sistema político-económico predominante ante los desafíos que lleva consigo la gestión de crear un orden global democrático. La salud es parte del problema y constituye un desafío sui géneris.

\section{¿La salud en crisis?}

La crisis afecta la salud en virtud de su impacto negativo sobre la vida cotidiana, por las condiciones laborales y sociales, el desempleo y los bajos ingresos, la inseguridad social, la pobreza... Precisamente, en estas condiciones, cuando más se necesita que la medicina ofrezca un servicio accesible y sostenible, sufre restricciones económicas; mientras las ganancias de los proveedores y la exclusión social se incrementan y agravan la vulnerabilidad de la salud. 
En el fondo, esa vulnerabilidad no sorprende. Los sistemas de salud modernos necesitan de un sólido financiamiento para el mantenimiento y desarrollo de la tecnología médica, el material sofisticado que ella requiere y la formación de profesionales muy especializados. Así, en medio de la crisis, las facturaciones de la industria médico-farmacéutica y tecnológica están menos afectadas que aquellas de otros sectores.

Desde hace algún tiempo, la sanidad viene operando al borde de sus posibilidades financieras y ya no resiste un gravamen adicional. Las Ilamadas "reformas de salud", diseñadas para contener los gastos y mejorar la eficiencia, resultan decepcionantes. En lugar de estabilizar los servicios de salud, lo que ellas logran es la autoestabilización de su crisis en tanto que los gastos continúan creciendo.

\section{Desigualdad de acceso a los servicios médicos}

Una medicina cara conduce necesariamente a la desigualdad en el acceso a los servicios médicos. Ello no se corresponde con los valores morales, éticos, sociales, religiosos de ninguna cultura, ni con la Declaración de los Derechos Humanos de la Organización de las Naciones Unidas, para no mencionar el compromiso profesional humanitario de la medicina misma; pero además, ni siquiera se corresponde con los proclamados principios democráticos a los cuales gustan de referirse en los países desarrollados.

La desigualdad de acceso a los servicios médicos forma parte del conflicto fundamental de la época: la contradicción entre el dominio del mercado y el compromiso social consustancial a la verdadera democracia. Aún cuando pudieran establecerse equilibrios precarios entre el egoísmo económico y el bien común, el capitalismo como sistema se contrapone a los principios democráticos, ya que, mientras el objetivo del mercado es incrementar los beneficios económicos pasando por encima de las graves desigualdades que crea en las condiciones de vida, la verdadera democracia defiende la idea de la igualdad en la satisfacción de necesidades fundamentales y el disfrute de los derechos humanos.

Aún cuando la fluidez de los cambios de la economía mundial hace difícil hacer cálculos precisos y actualizados, la dimensión del problema puede entenderse mejor con una mirada a los gastos sanitarios. Más de 3 millones de millones de EUR se invertían en suministros médicos al nivel global en el $2004,{ }^{1}$ (lo que equivalía a tres veces los gastos en armamentos). Las tres cuartas partes de esos recursos estaban a disposición de los EE. UU., los Países Ricos que formaron inicialmente la Unión Europea (PRUE) y Japón, los cuales constituyen un total de menos de $14 \%$ de la población global, y solo una cuarta parte para el $86 \%$ restante de la humanidad. En el año 2009 los gastos sanitarios globales se acercaron a 4 millones de millones de EUR. Veamos una aproximación para ese año de los promedios de los gastos sanitarios per cápita y año (pca), los que no dicen nada sobre el acceso individual a los servicios médicos que se produce al interior de los países, pero sí dicen mucho sobre la desigualdad entre los diferentes grupos de países, la que sigue agravándose desde entonces (tabla). 
Tabla. Aproximación para el año 2009 de los gastos sanitarios per cápita

\begin{tabular}{|l|c|c|}
\hline & $\begin{array}{c}\text { Habitantes } \\
\text { (millones) }\end{array}$ & $\begin{array}{c}\text { Gastos sanitarios/per } \\
\text { cápita (EUR) }\end{array}$ \\
\hline EE. UU. (2008) & $\sim 300$ & 6000 \\
\hline PRUE* & $\sim 300$ & $2000->3000$ \\
\hline Países de bajo ingreso económico & $\sim 3000$ & $<300$ \\
\hline Países económicamente pobres & $\sim 3000$ & $<35$ \\
\hline
\end{tabular}

*Países Ricos Unión Europea.

Casi el noventa por ciento de la población global dispone de un promedio de gastos sanitarios de menos de EUR 300/(pca), la mitad de ellos de mucho menos, mientras que en EE. UU. se esperaba llegar a EUR 7 000/(pca), en 2010. ${ }^{3}$ La fórmula de "la comunidad de los pueblos" suena ajena ante tal contraste. ¿Hasta donde llegará la carrera de los gastos sanitarios? ¿Qué perspectiva tiene el compromiso de igualdad en la atención médica? ¿Pueden los sistemas de medicina y de sanidad contemporáneos cumplir con su compromiso? Realicemos unos sencillos cálculos.

Para lograr la igualdad global en la atención médica, tomando como referencia los gastos actuales de EE. UU., se requerirían casi 50 millones de millones de EUR, lo que equivale el producto interno bruto (PIB) actual de todos los países del globo. $\mathrm{Ni}$ siquiera tomando como base los gastos sanitarios europeos, siendo estos algo más bajos que aquellos en EE. UU., tampoco sería económicamente posible lograr la igualdad. La apuntada extrapolación es simplista, pasa por alto cambios derivados del desarrollo económico, demográfico, ambiental, científico-técnico y otros factores que podrían modificar las condiciones de vida, de la salud y la sanidad. No obstante, el mensaje esencial es cierto si partimos de que se mantenga vigente el compromiso con los derechos humanos y democráticos, así como los valores sociales y culturales que constituyen la condición humana. Ello demuestra que los sistemas actuales de sanidad de los países económicamente ricos no sirven para el servicio sanitario global. Las cifras en bruto muestran lo absurdo de la situación y muestran la necesidad de un cambio sustancial. Para lograrlo hay que conocer las causas reales de los altos costos.

\section{Mercado, Estado y la salud}

Por lo general se hace recaer la culpa de los altos costos solamente sobre el progreso científico-técnico y el desarrollo demográfico. Tal interpretación es tan superficial que no merece ser discutida. En la realidad, los altos y crecientes costos obedecen a dos causas interrelacionadas. La primera es la entrega al mercado de una misión que le es ajena. El mercado y la medicina se asientan sobre principios opuestos. Mientras que la misión médica es altruista por principio, la fuerza motriz del mercado es el egoísmo económico, moralmente legitimado en un marco que estimula el suministro material, aun cuando trate de evitar el fraude y la injusticia. Por el contrario, en lo que concierne a la salud se excluye por principio cualquier acción por mero interés económico. No obstante, en la actualidad la agresiva economía del mercado no se detiene ante ningún aspecto de la vida. Según los criterios del mercado, la medicina se muestra particularmente atractiva por llevar una demanda garantizada, lo que se ofrece para obtener altas ganancias. Esto, en

http://scielo.sld.cu 
cierto grado, promueve el progreso médico, pero tiene efectos desastrosos para los costos y la igualdad de acceso, hasta llegar incluso al abuso de necesidades vitales e intervenciones de indicación dudosa.

El Estado, inventado para defender los derechos elementales, decepciona tanto más cuanto más se encuentre bajo la influencia neoliberal porque se convierte en promotor de intereses económicos en vez de servir los intereses públicos. Tanto más mercado y menos Estado, tanto mayores son los gastos y mayor la desigualdad. En tal ambiente los costos sanitarios tienen que crecer inconteniblemente sin que esto necesariamente se refleje en un impacto tangible en el estado de la salud de la población. Así, por ej., los gastos de salud en Gran Bretaña y EE. UU. ascienden a 2000 y 7000 EUR/(pca), respectivamente, en Cuba a 300 , mientras que los indicadores poblacionales del estado de salud de los tres países se parecen.

La otra causa de los altos costos concierne al propio concepto de la medicina: ¿Qué se espera de la medicina, cuál es su responsabilidad? El progreso científico-técnico trajo consigo una confianza virtualmente ilimitada en soluciones curativas para cualquier problema que afectara a la salud, y así, la medicina moderna se concentra en medidas curativas. Los médicos en su gran mayoría sienten que su responsabilidad es ocuparse de diagnosticar y curar las enfermedades. Sí, la prevención también se considera importante, pero en la realidad resulta postergada al segundo plano.

Algunas de las causas de los altos y crecientes costos por la orientación unilateral curativa de la medicina moderna son concluyentes y a su manera "legítimas": no caben dudas de la importancia del compromiso curativo, ni de la necesidad del progreso en el diagnóstico de las enfermedades y el tratamiento de los enfermos, ni de la aplicación de estímulos apropiados, ni de un gran rigor en la vigilancia jurídica de la calidad de los servicios médicos. En cada caso, en la implementación de las medidas adecuadas queda un desafío permanente. Permanecen por resolver: 1 . El vínculo fatal de la medicina con el mercado, 2 . La falta de un concepto médico sostenible.

\section{Un concepto estratégico de la atención médica}

A partir de los innegables éxitos y experiencia obtenidos mediante medidas preventivas en la lucha contra las enfermedades transmisibles, la medicina volvió a concentrarse en sus tareas curativas, pasando virtualmente por alto el desafío epidemiológico más grande en la historia de la humanidad: las enfermedades crónicas no transmisibles (ECTN).

Las ECNT son responsables del $60 \%$ de las muertes en el mundo (OMS 2010). En los países económicamente ricos la tasa llega a más de $80 \%$. Se trata de enfermedades cardiovasculares, neoplásicas, metabólicas, respiratorias, gastrointestinales, músculo esqueléticas, psiquiátricas, entre otras, de causas complejas, progresión lenta, duración larga y poca probabilidad de regresión y curación completa. Una vez que estas enfermedades se han manifestado, requieren tratamiento de por vida. En el desarrollo de las ECNT se conjugan condiciones genéticas, epigenéticas, sociales, ambientales e individuales relativas al estilo de vida.

Confiando en su potencial terapéutico y en el progreso científico-técnico, la medicina moderna agota sus esfuerzos y recursos en actividades curativas cada vez más sofisticadas y caras. El sistema de pago de los servicios médicos y de 
estímulos para las innovaciones curativas, en combinación con altas ganancias de la industria médico-tecnológica y farmacéutica, favorecen la orientación curativa.

Las prescripciones jurídicas vigilan especialmente cualquier posible omisión, por lo que el énfasis se pone en la aplicación de toda acción terapéutica potencialmente curativa. Así, aunque sea solamente por autoprotección, los médicos agotan las posibilidades diagnósticas, terapéuticas y de rehabilitación hasta más allá de lo necesario.

Esta es la situación de la medicina contemporánea. La influencia del mercado, el enfoque patocéntrico y la alta morbilidad y mortalidad por ECNT causan un crecimiento de los costos sanitarios más alto que aquel del PIB. Hace mucho que los gastos sobrepasaron los límites críticos en los países económicamente ricos y siguen aumentando, mientras que la desigualdad en asistencia médica crece para la gran mayoría de la población hasta incluso en los propios países ricos. Con ello se agudiza el conflicto con los derechos humanos y los principios éticos de la propia medicina.

Las ECNT se ofrecen como punto de partida para reconsiderar los principios del proceder de la medicina moderna. En vez de seguir agotándose en reparar los daños a posteriori, lo que exige en demasía los recursos económicos y aumenta la desigualdad en correspondencia con intereses económicos inadecuados, lo que se requiere es fortalecer la salud y hacerla menos vulnerable a las ECNT. Esto precisa de un proceder estratégico.

La lección clave de las enfermedades transmisibles, la cual nos enseña que se requiere de un proceder estratégico para vencer una epidemia producida por un agente patógeno determinado, se hace compleja cuando se trata de las ECNT. En este caso no se trata de un solo agente patógeno, ya que en estas enfermedades la etiología y la patogénesis requieren la consideración de muchos factores e interacciones de la vida cotidiana, así como múltiples condiciones sociales y ambientales. A la luz de las diversas condiciones que no contribuyen al mantenimiento de la salud, es indispensable fortalecer el enfoque proactivo sanocéntrico en vez de solamente defenderse mediante actividades patocéntricas específicas.

Una de las virtudes de la profesión médica es la capacidad de reaccionar rápido y adecuadamente en defensa de la salud cuando se presenta la enfermedad. Combatir una epidemia, sin embargo, requiere acciones anticipadas, medidas que adoptar antes que se presente una necesidad inmediata. En el caso de las ECNT hay que accionar con anticipación excepcional y de manera muy cautelosa por la gran complejidad etiológica y progresión lenta, hasta alcanzar una cultura salutogénica. El cambio de pensamiento y actitud necesario es considerable y nada fácil de realizar, como lo muestra el actual desamparo ante las ECNT.

Acostumbrada al éxito del proceder reactivo, a la medicina actual le resulta difícil comprometerse con un concepto proactivo, dirigido a fines futuros y adoptar una estrategia a largo plazo para llegar finalmente al objetivo. La reorientación ahora, sin embargo, es imprescindible para darle sostenibilidad a la medicina moderna y disminuir la desigualdad que produce. Se hace necesario un concepto estratégico de reducción del riesgo de enfermedad mediante la promoción de la salud, al que se incorporen las actividades curativas que se hagan necesarias. De otra manera no será posible afrontar con éxito el desafío planteado por las ECNT, con todas sus consecuencias humanitarias, económicas y sociales.

Un concepto estratégico sanocéntrico requiere mejores conocimientos acerca de la salud y de la relación dinámica entre salud y enfermedad. La medicina 
contemporánea sabe mucho de las enfermedades y la patogénesis, pero poco de la salud y la salutogénesis. A pesar de la obvia dependencia que tiene la salud de múltiples factores individuales, sociales y otros, la medicina moderna se limita por lo general a los mecanismos biológicos y dedica poca atención a los demás factores etiológicos y patogénicos y menos aún a las condiciones y los determinantes de una buena salud.

\section{Los determinantes individuales y sociales de la salud}

Hace más de 150 años Rudolf Virchow y Friedrich Engels señalaron la influencia de las condiciones sociales de vida sobre el estado de salud y la resistencia contra las enfermedades. En 2003 la OMS llamó la atención sobre el problema en una publicación clave, ${ }^{4}$ en 2005 estableció la "Comisión de Determinantes Sociales de la Salud", la cual presentó un informe clave en $2008,{ }^{5}$ otro en $2010 .{ }^{6}$ A pesar de su larga historia, el conocimiento de los determinantes sociales de la salud tiene poca repercusión en la práctica médica. La medicina sigue concentrándose en medidas curativas en un ambiente dominado por el mercado donde hasta los servicios médicos tienden a ser tratados como mercancía (el enfermo, es quien trae el dinero). En tales condiciones, la atracción económica de una medicina que se esfuerce en reducir la morbilidad mediante diferentes intervenciones a nivel social no es muy alta. No obstante, el desafío de las ECNT no le deja alternativa a la medicina que incorporar los determinantes sociales en el espectro de sus actividades. Además de los determinantes sociales, tienen primordial importancia los determinantes individuales de la salud. Al conjunto de todos ellos les llamamos Determinantes Individuales y Sociales de la Salud (DISS).

La disciplina médica más apta para ocuparse de los DISS, en virtud de su pensamiento integral avanzado y de su mayor penetración en el ambiente social, es la Medicina General Integral tal como la que se practica en Cuba. ${ }^{7}$ Corresponde a esta medicina marcar el paso de manera idónea a fin de lograr la integración de los DISS en una medicina estratégica sanocéntrica anticipativa, apta para enfrentar los desafíos médicos del siglo XXI.

\section{UNA INICIATIVA: DISSFA}

Aprovechando la vocación preventiva de la medicina cubana y su apropiado sistema de salud, se lanzó un proyecto en un policlínico del Centro Histórico de La Habana con el objetivo de sensibilizar a un grupo de médicos de la familia sobre la necesidad de establecer entre estos y con cada uno de los individuos de la comunidad que atienden, una comunicación aún más amplia, minuciosa, sistemática, personalizada y dirigida hacia los DISS. El proyecto "Determinantes Individuales y Sociales de la Salud en la Medicina Familiar" (DISSFA), aspira a determinar la factibilidad y el impacto en la salud de tal comunicación en las consultas médicas, sin descuido de las tareas curativas. En "consultas salutogénicas" se registran los temas enfocados, el tiempo y la intensidad de comunicación, así como la receptividad, el impacto en el estilo de vida y el estado de la salud a mediano y largo plazo. El proyecto DISSFA pretende ser un impulso práctico para una Medicina Estratégica Sanocéntrica Anticipativa, capaz de proyectarse también hacia otros países que pretendan salir de la crisis médica mediante un concepto sostenible. Al mismo tiempo el proyecto DISSFA constituye un homenaje al renacimiento de La Habana Vieja, bella y querida. Quiere ser una modesta contribución a un plan humanitario de gran empeño y agregar algunos logros tangibles a esa elevada visión, en bien de los ciudadanos allí residentes y como un modelo para su aplicación más allá en el futuro. 


\section{REFERENCIAS BIBLIOGRÁFICAS}

1. Word Health Organization. Spending on Health, Fact sheet $N^{\circ} 319$. Geneva: WHO; 2007.

2. U.S. Health Care Costs, National Health Expenditures. Washington, D.C.: Kaiser Family Foundation; 2008

3. Duncan DE. What Price for Medical Miracles? [Internet]. 2010 [citado 22 Ago 2009]. Disponible en: http://www.kaiserhealthnews.org/Stories/2010/March 109/fiscal-times-end-of-life.aspx

4. Wilkinson R, Marmot M. editor. Social Determinants of Health-The Solid Facts. Geneva: WHO, Regional Office for Europe, Copenhagen; 2003.

5. Word Health Organization. Commission on Social Determinants of Health. Final Report: Closing the Gap in a Generation, Health Equity through Action on Social Determinants of Health. Geneva: WHO; 2008.

6. Word Health Organization. Action on the Social Determinants of Health: Learning from Previous Experiences. Geneva: WHO; 2010.

7. Thielmann K. Determinantes de Salud: potencial investigativo y estratégico de la Medicina General Integral. Rev Cubana Med Gen Integr [Internet]. 2005 [citado 22 Ago 2009];21(5-6). Disponible en:

http://scielo.sld.cu/scielo.php?script=sci arttext\&pid=S0864 -

$\underline{21252005000500021 \& \operatorname{lng}=e s \& n r m=i s o \& t \mid n g=e s}$

Recibido: 1 de noviembre de 2011.

Aprobado: 12 de enero de 2012.

José IIInait Ferrer. Centro Nacional de Investigaciones Científicas. Calle 158 y Ave. 25, Playa. La Habana, Cuba.

Telf.: 208 5834. Correo electrónico: jose.illnait@cnic.edu.cu

http://scielo.sld.cu 